Check for updates

Cite this as: $B M J 2022 ; 376: 0604$ http://dx.doi.org/10.1136/bmj.0604 Published: 08 March 2022

\section{Which antihypertensive treatment is better for mild to moderate hypertension in pregnancy?}

In this article by Danielle Ashworth and colleagues (BMJ 2022;376:e066333; doi:), the definition of mild to moderate pregnancy hypertension (given in the last paragraph of the introduction) was incorrect; it should have been defined as systolic blood pressure 140-159 mm Hg and/or diastolic blood pressure 90-109 mm Hg. The online article and PDF have been corrected. 